\title{
Mortality after admission to hospital with fractured neck of femur: database study
}

\author{
Michael J Goldacre, Stephen E Roberts, David Yeates
}

Unit of Health-Care

Epidemiology,

Department of

Public Health,

Institute of Health

Sciences, University

of Oxford, Oxford

OX3 7LF

Michael J Goldacre

director

Stephen E Roberts

statistician

David Yeates

computer scientist

Correspondence to:

M J Goldacre

michael.goldacre@

dphpc.ox.ac.uk

BMJ 2002;325:868-9

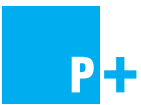

A table with further data is available on bmj.com
The death rate within one year of fractured neck of femur is typically reported as between $20 \%$ and $35 \%$. $^{1-3}$ Performance indicators based on mortality after hospital admission for such fractures have been promoted. ${ }^{4}$ The only measure of mortality in routine hospital statistics, however, is "in-hospital mortality"-death during the initial admission for the fracture. We analysed inpatient statistics that had been linked to death registration data in the former Oxford NHS health region (population 2.5 million) from 1994 to 1998.

\section{Methods and results}

We selected emergency admissions in people aged 65 years and over who had been admitted to eight main acute trusts and for whom fractured neck of femur was the principal diagnosis (international classification of diseases, ninth revision (ICD-9), codes 820, 821.0, and 821.1). We calculated standardised mortality ratios after fracture by applying the age and sex specific mortality in five-year age groups in the whole population of the region ("standard" population) to the number of people with fractured neck of femur in the equivalent age and sex strata, in successive months up to one year after fracture. For each hospital, we calculated case fatality rates for in-hospital deaths within 30 days and for all deaths within 30, 90, and 180 days of admission. We calculated age and sex standardised case fatality rates for each hospital by applying the age and sex specific rates in each hospital to the number of people in each age-sex stratum in the total inpatient population. We calculated case fatality rates separately for deaths certified as fractured femur and for all deaths.

In total, 8148 people aged 65 and over were included $(80.2 \%$ women; mean (SD 7.2) age 82.2 years). In the first month after fracture the standardised mortality ratio was 1246 (95\% confidence interval 1164 to 1331; general population 100). The standardised mortality ratios, adjusted for person months at risk, were 451 (397 to 509) in month 3, 238 (197 to 283 ) in month 6 , and 187 (149 to 230 ) in month 12. Fractured femur was certified as the underlying cause in $16 \%$, and as a cause anywhere on the death certificate in $43 \%$, of deaths occurring in the first month.

As the table shows, the mortality ranking of hospitals varied with definitions and time frames. Death rates for all causes showed that three hospitals $(\mathrm{B}, \mathrm{C}$, and $\mathrm{H})$ had significantly lower rates than hospital $\mathrm{A}$ for in-hospital mortality within 30 days, and two (B and C) had significantly lower rates for 30 day mortality regardless of place of death. By 90 and 180 days, differences between hospitals were not significant. Hospitals also changed rank depending on whether deaths from all causes or only those certified as fractured femur were included (table).

\section{Comment}

The standardised mortality ratios show that mortality is much higher in people after fractured neck of femur than in the general population of comparable age, and they remain raised for many months after fracture. The

Case fatality rates per 100 admissions and ranks (95\% confidence intervals) for each hospital, adjusted for age and sex, showing different definitions and time intervals from admission

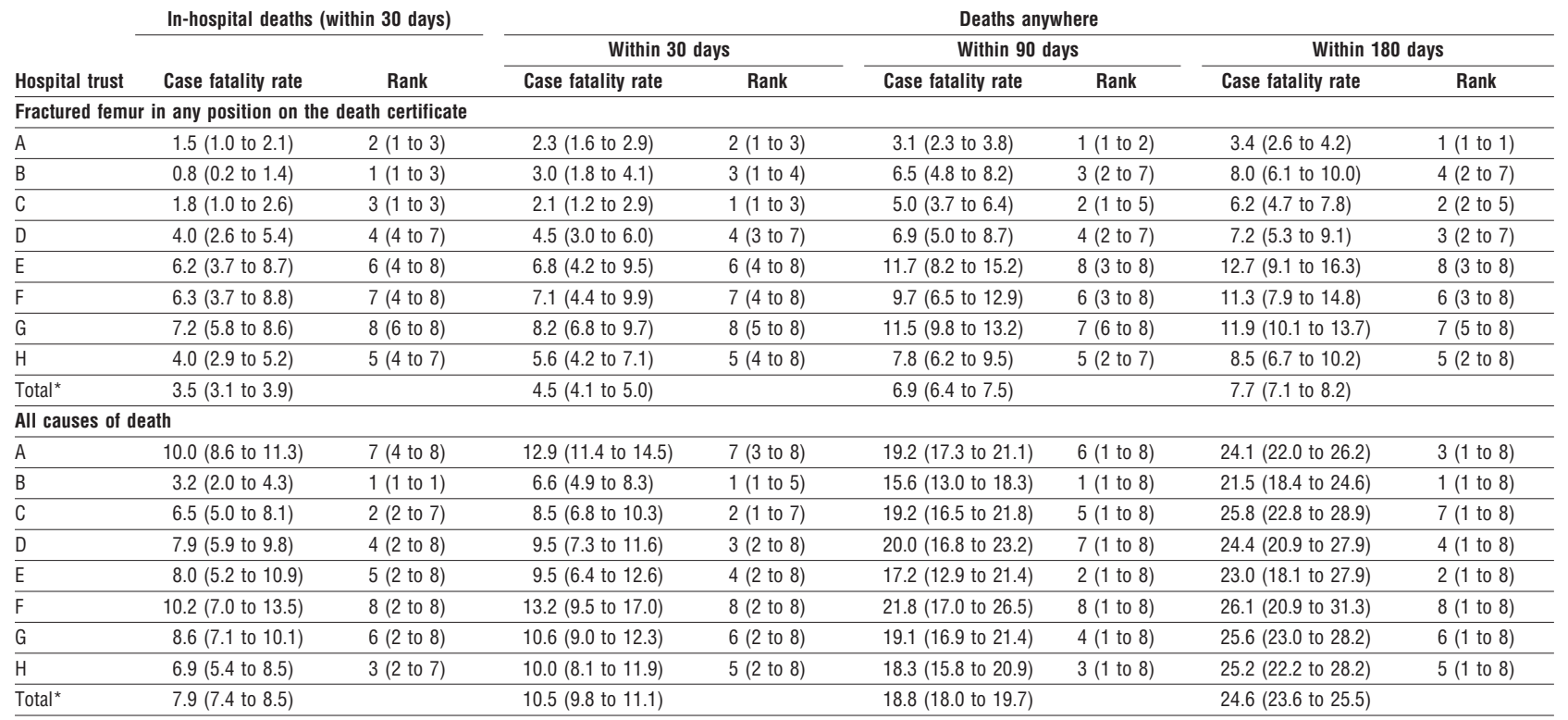

${ }^{\star}$ Total case fatality rates are unadjusted for age and sex. 
persistently increased standardised mortality ratio may indicate continuing sequelae of the fracture or that people fracturing their neck of femur are more frail and ill than the general population of similar age.

Measures of prognosis after fracture and comparisons between hospitals are substantially affected by whether death registration data are included, whether time intervals are extended beyond 30 days, and whether deaths that are not certified as fractured femur are included. When death registration data are available, one option is to confine analyses of mortality to the deaths attributed by the certifying clinician to the fracture. Our study confirms, however, that the fracture is often not recorded on death certificates even when death occurs soon after fracture. ${ }^{5}$ Studies of mortality after fractured femur will be misleading unless they include deaths after discharge from the initial admission and consider all causes of death.

We thank Pamela Evans for preparing the manuscript.
Contributors: MJG designed the study and jointly wrote the manuscript. SER contributed to the design, analysed the data, and jointly wrote the manuscript. DY extracted the data, contributed to the design, and commented on the manuscript. MJG and SER are guarantors for the paper.

Funding: SER receives funding from the Department of Health (the views expressed in this paper are those of the authors and not necessarily those of the Department of Health). The Unit of Health-Care Epidemiology is funded by the South East regional office of the NHS Executive.

Competing interests: None declared.

1 Todd CJ, Freeman CJ, Camilleri-Ferrante C, Palmer CR, Hyder A, Laxton CE, et al. Differences in mortality after fracture of hip: the East Anglian audit. BMJ 1995;310:904-8

2 Keene GS, Parker MJ, Pryor GA. Mortality and morbidity after hip fractures. BMJ 1993;307:1248-50

3 Boereboom FT, Raymakers JA, Duursma SA. Mortality and causes of death after hip fractures in the Netherlands. Neth J Med 1992;41:4-10.

4 NHS Executive. Quality and performance in the NHS: clinical indicators: Leeds: NHSE, 1999:30-7.

5 Goldacre MJ. Cause-specific mortality: understanding uncertain tips of the disease iceberg. J Epidemiol Community Health 1993;47:491-6.

(Accepted 21 March 2002)

\section{Drug points}

\section{Tonic-clonic seizures in patients taking sildenafil}

Ronit Gilad, Yair Lampl, Yehiel Eshel, Menachem Sadeh, Department of Neurology, Wolfson Medical Center, Holon 58100, Israel Correspondence to: R Gilad (gilad-ar@inter.net.il)

Sildenafil citrate (Viagra) is a specific phosphodiesterase type $\mathrm{V}$ inhibitor with a selective inhibitory effect on cyclic guanosine monophosphate (cGMP). Its enhancement of nitric oxide release leads to an increase in cGMP concentrations, which is responsible for its main clinical effect-relaxation of the smooth muscle in the corpus cavernosum. The increase in blood flow into the penis helps to alleviate problems of erectile dysfunction.

Minor side effects-such as headache, flushing, nasal congestion, and defects in colour vision-and serious cardiovascular and cerebrovascular effects, have been described. ${ }^{1}$ Convulsive seizures have been an unknown neurological side effect until now. We report two cases of patients who first had a seizure soon after using sildenafil.

Case 1-A 63 year old man with a history of hypertension, but who was otherwise healthy, was admitted to hospital because of a first episode of generalised tonic-clonic seizures (GTCS). He had been prescribed sildenafil $(50 \mathrm{mg})$ as required for sexual dysfunction. Three hours after taking sildenafil for the first time, he had a typical tonic-clonic seizure, as observed by his spouse. He was awake when he was admitted, and results of neurological examination, brain computed tomography, magnetic resonance imaging, and electroencephalography were normal. Electrocardiography, stress electrocardiography, echocardiography and cardiac scan with dipyridamole test, as well as carotid Doppler ultrasonography, showed no abnormalities. Two days later, he was discharged from the hospital with the recommendation that he stop using sildenafil. However, three months later, he decided on his own responsibility to try the drug again, and four hours later, had a tonic-clonic seizure. He was then prescribed carbamazepine (600 mg/day). He has been free of seizures for two years.

Case 2-An otherwise healthy 54 year old man was admitted to our department after having a first tonic-clonic seizure about 4.5 hours after he had taken sildenafil for the first time. His neurological examination, brain computed tomography, magnetic resonance imaging, and electroencephalography with sleep deprivation were normal.

\section{Discussion}

These men experienced epileptic seizures after taking sildenafil. It is highly unlikely that the seizures were caused by a cerebrovascular effect-that is, a previous existing neurological disease- or a cardiac event because medical tests ruled out this possibility, and in both cases the seizures occurred after the physical effort associated with coital activities had ended. Using sildenafil and having seizures seem to be directly related.

These two cases raise two important issues. Firstly, doctors must consider the possibility of epileptic seizures occurring in patients taking sildenafil. Secondly, more research is needed to test two conflicting theories-that is, whether nitric oxide induces epilepsy or protects against it.

Four cases of patients experiencing epileptic seizures during the clinical trials of sildenafil were reported to Pfizer, the manufacturer of the drug. However, a good correlation between the seizures and the effects of the drug was not found.

Funding: None.

Competing interests: None declared.

1 Moreira SG, Brannigan RE Jr, Spitz A, Orejuela FJ, Lipshultz LI, Kim ED. Side-effect profile of sildenafil citrate (Viagra) in clinical practice. Urology 2000;56:474-6

\section{Endpiece}

\section{A gloomy thought}

Bodily decay is gloomy in prospect, but of all human contemplations the most abhorrent is body without mind.

Thomas Jefferson (1743-1826) in a letter to John Adams, 1 August 1816.

In Familiar Medical Quotations, ed Maurice B Strauss, Little, Brown and Company, Boston, 1968

Submitted by Fred Charatan, retired geriatric physician, Florida 